\title{
THE ENUMERATION AND MEASUREMENT OF FAT GLOBULES IN MILK.
}

By W. F. Cooper, B.A. (Cantab.), F.C.S., W. H. Nutrall, F.I.C., F.C.S., AND G. A. Freak, B.Sc., A.I.C.

The Cooper Laboratory for Economic Research, Watford.

IN investigating the influence of various factors on the character of milk, it is now recoguised that it is insufficient (at least for many purposes) to make comparisons of the percentage of fat, but some regard must be paid to the distribution of the fat in the globules. The technique of such investigations is somewhat complicated, and, as it was impossible to include in our previous papers a full description of the many points involved, it has been thought desirable to give the working details of the method employed, and at the same time to compare it with the method employed by previous workers.

It is hoped that these details may prove of considerable assistance to subsequent workers on similar problems.

Broadly speaking, there are two general methods for the enumeration and measurement of fat globules in milk; in one, the milk is examined in a capillary tube; in the other, in a flat cell. Both these methods were originally devised for the counting of corpuscles in blood, and have been adopted, with slight modifications, for milk examinations.

The capillary tube method was introduced for milk examinations by Bouchut, but, as Babcock made many extensive investigations with it, the method is usually associated with his name.

Babcock's original paper (Fourth Annual Report, N.Y. Expt. Station, Geneva, 1886, pp. 226, 275) is not generally available. Gutzeit, who has employed Babcock's method in a very exhaustive investigation on milk globules, has published, with his results, a full description of the method (Landwirtschaftliche Jahrbücher, 1895, 545). Since this journal is not easily accessible in this country, the authors 
have made a detailed abstract of Gutzeit's description of Babcock's method.

The present authors have employed the flat cell method, with certain modifications, as it appears to present distinct advantages ver that of Babcock.

\section{Babcock's Method of Investigation.}

Detailed Abstruct from Gutzeit.

In order to measure the mean diameter of fat globules by Babcock's method, the number of globules in a measured volume of milk is determined. For this purpose, glass capillaries, of about $0.1 \mathrm{~mm}$. diameter and $2-3 \mathrm{cms}$. Iong, are employed, the diameter being as constant as possible over small lengths.

$10 \mathrm{ccs}$. of milk are diluted in a graduated flask to $500 \mathrm{ccs}$. With rich milk, or towards the end of the period of lactation, 5 ccs. may be used.

The capillary tubes are filled by dipping the end into the well mixed diluted milk, and the ends of the tubes are then sealed by melting in a small flame. The tubes are placed side by side, on a microscope slide, and fixed at both ends with a drop of melted wax. The slide is allowed to remain for half an hour on the microscope stage, which must be perfectly horizontal; a drop of water (or glycerine) is placed on the tubes, which are then covered with the cover-slip. By using an objective of sufficient magnification, the fat globules can be clearly recognised and counted through the capillary wall.

If the inner transverse diameter of the capillary is read off in terms of the divisions of the ocular micrometer, the ocular scale being at right angles to the length of the tube, and the micrometer focussed on the two bounding lines, the volume in which the enumerated globules are suspended can easily be calculated.

As we shall see later, the two lines do not represent the actual limits of the capillary walls, though they bear a definite relationship to them; their relative position is not affected, if the convergency of the illuminating rays (aperture of the sub-stage condenser) is altered, as would be the case if it were an interference phenomenon. Figure 1 shows the appearance of such a tube under the nicroscope at a magnification of 400 diameters, with an image of the ocular micrometer superposed. 
W. F. Cooper, W. H. Nuttall and G. A. Freak 359

A tube, 100 micrometer divisions long and 100 divisions broad, is taken as a unit tube; and the number of globules which would occur in a volume of milk required to fill such a tube is calculated by multiplying by $100^{2}$ the number of fat globules found in a capillary 100 divisions long, and dividing by the square of the diameter of the capillary, measured in micrometer divisions.

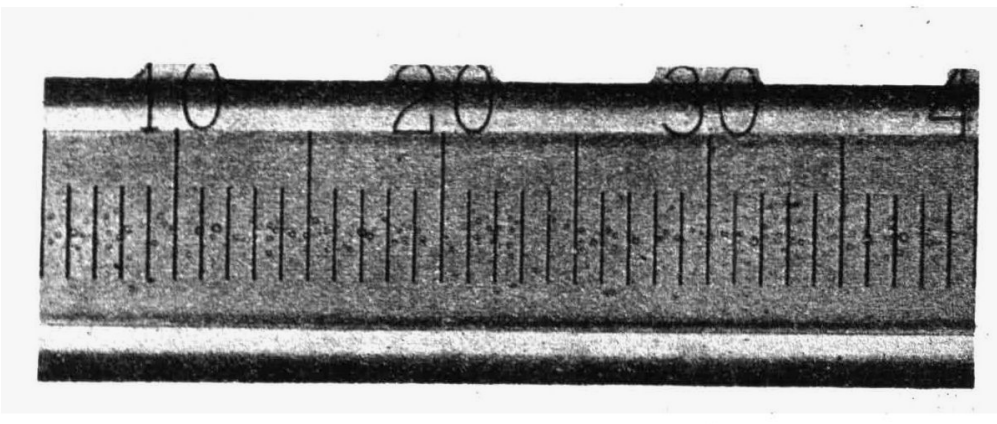

Fig. 1.

Thus, if $a$ represents the number of enumerated globules and $d$ the internal diameter of the tube, the number of globules occurring in a unit tube is given by the formula $\frac{a \times 10,000}{d^{2}}$, and, provided investigations are always carried out at the same dilution and magnification, the various values given by this formula may be directly compared.

In Gutzeit's experiments, one micrometer division corresponded to $0.0025 \mathrm{~mm}$.; in Babcock's and Woll's work, to $0.0024 \mathrm{~mm}$.

If $d$ represents the internal diameter of the tube-measured in micrometer divisions-and the length of the tube always corresponds to 100 divisions, then the volume $(J)$ of the tube used for each measurement is given by the equation

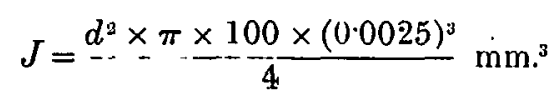

If, in this volume, $a$ globules are counted, then $0.0001 \mathrm{~mm} .^{3}$ of the diluted milk will contain

$$
\frac{a \times 4 \times 0.0001}{d^{2} \times \pi \times 100 \times(0.0025)^{3}} \text { fat globules, }
$$

Journ. of Agric. Sci. $v$ 
and, since the milk has been diluted 50 times, $0.0001 \mathrm{~mm} .^{3}$ of milk will contain

$$
\frac{a \times 4 \times 0.0001 \times 50}{d^{2} \times \pi \times 100 \times(0.0025)^{3}}=\frac{a \times 4074}{d^{2}}
$$

fat globules. $0.0001 \mathrm{~mm}^{3}$ is taken as the "standard quantity," and the number of fat globules occurring in this quantity is designated by $N$. Thus

$$
N=\frac{a \times 407+}{d^{2}} .
$$

In order to decrease the error due to the uneven distribution of the fat globules, 3 counts on each tube were made, thus 9 counts in all ; 15 in some very careful estimations (Babcock and Woll made 5 counts on each of 3 tubes, but their micrometer scale was only half as long as Gutzeit's).

An example is given below.

Montavon Cow "Rezia." 20th August.

Fat content $3.22 \%$. Mill diluted 50 times.

\begin{tabular}{c|c|c|c}
\hline Capillary No. & I & II & III \\
\hline$d=$ & 47 & 49 & 47 \\
\hline 1st reading $a=$ & 12.4 & 150 & 177 \\
2nd reading $a=$ & 190 & 170 & 153 \\
3rd reading $a=$ & 183 & 194 & 166 \\
\hline Total & 497 & 514 & 496 \\
\hline Mean & 166 & 171 & 165 \\
\hline
\end{tabular}

Using the formula previously given, $\frac{a \times 10,000}{d^{2}}$, these readings give 737 as the mean figure; that is to say, there are 737 fat globules contained in a unit tube. This number multiplied by the factor 0.4074 , obtained above, gives 300 as the number of fat globules $(N)$ occurring in $0.0001 \mathrm{~mm}^{3}$ of milk. Further, if the fat content of the milk $(=f)$ is known, there are the following relationships.

In $0.0001 \mathrm{~mm}^{3}$ of milk there are $\frac{0.0001 \times f}{100} \mathrm{~mm}^{3}$ of fat. This quantity of fat corresponds to the quantity in $N$ globules. The volume 
of the mean size globule is, therefore, $\frac{0.0001 f}{100 N}=0.000,001 \times \frac{f}{N} \mathrm{~mm}^{3}$ This expression represents Babcock's "relative size." In the example given $f=3.22 \%, N=300$, the relative size

$$
=\frac{3 \cdot 22}{300} \times 0.000,001 \mathrm{~mm} \cdot{ }^{3}=0.000,000,0107 \mathrm{~mm} .^{3}
$$

As Babcock himself pointed out, this expression does not give absolute results, since the fat content is expressed in weight percentage, and no allowance for the specific gravity has been made. If the mean specific gravity of milk is taken as 1.0315 , and that of butter fat as 0.94 at $15^{\circ}$, the volume of the mean size globule $(V d)$ is obtained by multiplying the value for the relative size by $\frac{1 \cdot 0315}{0.94}=1 \cdot 1$. This figure is used as a constant throughout this work.

In the above example $\nabla d=0 \cdot 000,000,0107 \times 1 \cdot 1=0.000,000,0118 \mathrm{~mm}^{3}$ $\mathrm{F}$. om the mean volume, the mean diameter of the globules can be calculated, but as all variations in the mean size are more evident when expressed as volumes, Gutieit, like Babcock and Woll, compared the mean volumes, and only exceptionally calculated the mean diameters.

In order to be able to deal better with the small numbers involved, the micromillimetre $(=\mu=0.001 \mathrm{~mm}$.) was taken as unit of length, and $\mu^{3}$ as unit of volume. $V d$ in the above example $=11.8 \mu^{3}$, the "standard quantity" for the count of globules $=100,000 \mu^{3}$, the division of the micrometer scale $=2 \cdot 5 \mu$, etc.

To obtuin a clear image of the tubes, it is necessary to mount them in a liquid, as described; for this purpose Babcock and Woll used concentrated glycerine. The value for $d$ varies according to the liquid used; the same tube for instance having an apparent diameter of 65 micrometer divisions in air, 56 in water, and 52 in glycerine. This phenomenon is due to the refraction of the light from the glass into the less dense liquid. Allowance can be made for it by dividing the diameter of the tube, read off on the micrometer, by the refractive index of the glass, of which the tube is composed, compared with that of the mounting liquid. If the mounting liquid, for instance, Canada Balsam, has the same refractive index as the glass, the measurements given by the microscope represent the real diameter of the tube. As a matter of fact, Gutzeit found it more convenient to mount the capillary tube in water, the index of refraction always being 1.333 , and to calculate the value for $d$. For this purpose, the index of refraction 
of the glass used was taken as identical with that of Canada Balsam, which was found, by means of an Abbe refractometer, to be 1.514; the refractive index of glycerine is 1451 . Therefore the factor required to convert $d$ to an absolute value is $\frac{1.333}{1.514}=0.88$ if water is used, and $\frac{1.451}{1.514}=0.96$ if glycerine is used. These factors may be obtained without the use of a refractometer, by measuring the same tube in Canada Balsam and in water.

$$
\begin{array}{rlr}
d \text { in Cauada Balsam } & =50.3 & 50.3 \\
d \text { in water } & =56.0 & \frac{56.0}{56 .}=89 .
\end{array}
$$

If absolute values are required, the diameter of the capillary having been determined in water or glycerine, $N$ (the number of fut globules) must be divided by the square of the corresponding factor $(0.88$ in the case of water and 0.96 in the case of glycerine), and $V d$ (the mean volume) multiplied by it.

For instance, in the example previously given, $a$ having been determined in water (as is the case throughout this work), the absolute value of

$$
V d=11.8 \times 0.89^{2}=9.51 \mu^{3} .
$$

It is obvious from the foregoing that, theoretically, Babcock's method, with the slight modifications introduced, is reliable, and should give absolute values. Woll's parallel estimations with it agree well, differing by only a few per cent. (Agric. Science, 1892, 445).

Considering the examples given above; it will be noticed that the readings in the same tube differ by about $30 \%$; this, however, is not surprising, as, at a dilution of 50 or 100 times, a constant and equal distribution of the fat globules is not possible. The three figures which are obtained from the formula $\frac{N \times 10,000}{d^{2}}$, viz. 752, 712, 747, agree within $6 \%$; in the worst cases this difference amounted to $10 \%$. If we take two complete estimations with three capillaries and five readings on each, we find, for instance:

4th January, 1893. Milk from Municipal Stall. $f=4.51 \%$.

$$
\begin{aligned}
& \text { 1st experiment } N=488, V d=10 \cdot 19 \mu^{3} \text {. } \\
& \text { 2nd experiment } N=501, V d=9 \cdot 90 \mu^{3} \text {. } \\
& \text { Difference } 13 \quad 0.3 \mu^{3} \text {. }
\end{aligned}
$$

The difference between the two values for $N$ is 13 globules; that between the two values for $V d, 0.3 \mu^{3}$, or only about $3 \%$. Repeated 


\section{W. F. Cooper, W. H. Nuttall and G. A. Freak 363}

estimations gave similar results. Better results were usually obtained with a smaller fat content and a higher value for $V d$.

More accurate values for $V d$ may be obtained, if desired, by employing a greater magnification and a more accurate measurement of $d$ and by an increased number of counts on more than three tubes. As, however, the estimation of the fat, by means of the centrifuge, is only accurate to about $3 \%$ (calculated on the fat) even when the greatest care is taken, there is not much point in obtaining a more accurate figure for $d$. An idea of the accuracy of the value for $V d$, as given by this method, may be obtained by calculating the mean diameter $d$ from the formula $V=\frac{4}{3} \pi r^{3}$.

$$
\begin{array}{cc}
\text { Estimation 1. } & d=2.69 \mu . \\
" \quad 2 . & \frac{d=2.66 \mu .}{0.03 \mu} . \\
\text { Difference } &
\end{array}
$$

Since it is impossible to measure beyond $0 \cdot 1 \mu$, this difference is of no account.

In this connection, it may be mentioned that $V d$ for the same cow, within one lactation period, may vary as much as $300 \%$ from day to day.

Theoretically, a slight error might be introduced in the factor $1 \cdot 1$, as only mean values for the specific gravity of milk and for butter fat have been taken. The specific gravity of the milk could be determined easily in each case; that of the butter fat at $15^{\circ} \mathrm{C}$. not readily. If, however, experiments are carried out over the whole period of lactation, such variations would be eliminated in the mean $V d$.

The computation of the two values $N$ and $V d$ is simpler than it appears. If, instead of reducing the value of $d$, measured in water, to an absolute figure, we divide the final factor by $0.89^{2}$, we have

$$
N=\frac{a}{0.89^{2} \times d^{2}} \times 0.4074=\frac{a \times 0.5053}{d^{2}} .
$$

Further, when the same number of rearings are made with each of the three tubes, the divisions are saved, if the final factor is divided by $3 \times 3(=9)$ for three readings, $3 \times 5(=15)$ for five readings, and so on.

The following table has been constructed to indicate the constant, dependent upon the dilution of the rnilk, the number of tubes and the number of readings on each tube required in the foregoing equation. 


\begin{tabular}{|c|c|c|c|c|}
\hline $\begin{array}{c}\text { No. of } \\
\text { readings } \\
\text { for each tabe }\end{array}$ & Dilution & No. of tubes & $F$ & $\log F$ \\
\hline 3 & $\left\{\begin{array}{l}1: 50 \\
1: 100 \\
1: 50 \\
1: 100\end{array}\right.$ & $\begin{array}{l}2 \\
2 \\
3 \\
3\end{array}$ & $\begin{array}{l}\cdot 8422 \\
\cdot 1684 \\
\cdot 5614 \\
\cdot 1123\end{array}$ & $\begin{array}{l}.9254 \\
.2263 \\
\cdot 7493 \\
.0504\end{array}$ \\
\hline 5 & $\left\{\begin{array}{l}1: 50 \\
1: 100 \\
1: 50 \\
1: 100\end{array}\right.$ & $\begin{array}{l}2 \\
2 \\
3 \\
3\end{array}$ & $\begin{array}{r}\cdot 5053 \\
\cdot 1010 \\
\cdot 3374 \\
\cdot 6748\end{array}$ & $\begin{array}{l}\cdot 7035 \\
\cdot 0048 \\
\cdot 3374 \\
\cdot 8291\end{array}$ \\
\hline
\end{tabular}

In addition, to obviate the three divisions by $d^{2}$, the following table was used, in which, for each value of $d$, measured in micrometer divisions, the tubes being mounted in water, the value for $10-2 \log d$ can be read off.

\begin{tabular}{|c|c|c|c|c|c|c|c|c|c|}
\hline$d$ & $\begin{array}{c}10-2 \\
\log d\end{array}$ & $d$ & $\begin{array}{c}10-2 \\
\log d\end{array}$ & $a$ & $\begin{array}{c}10-2 \\
\log d\end{array}$ & $d$ & $\begin{array}{r}10-2 \\
\log d\end{array}$ & $d$ & $\begin{array}{c}10-2 \\
\log d\end{array}$ \\
\hline 30 & 0458 & 40 & 7950 & 50 & 6020 & 60 & 4437 & 70 & 3098 \\
\hline 31 & 0172 & 41 & 7744 & 51 & 5849 & 61 & 4293 & 71 & 2974 \\
\hline 32 & 9897 & 42 & 7535 & 52 & 5670 & 62 & 4157 & 72 & 2853 \\
\hline 33 & 9630 & 43 & 7332 & 53 & 5515 & 63 & 4013 & 73 & 2743 \\
\hline 34 & 9371 & 44 & 7132 & 54 & 5352 & 64 & 3876 & 74 & 2615 \\
\hline 35 & 9112 & 45 & 6936 & 55 & 5192 & 65 & 3742 & 75 & 2499 \\
\hline 36 & 8873 & 46 & 6746 & 56 & 5036 & 66 & 3609 & 76 & 2384 \\
\hline 37 & 8635 & 47 & 6558 & 57 & 4884 & 67 & 3478 & 77 & $2271^{\circ}$ \\
\hline 38 & 8404 & 48 & 6375 & 58 & 4731 & 68 & 3349 & 78 & 2158 \\
\hline 39 & 8179 & 49 & 6196 & 59 & 4583 & 69 & 3223 & 79 & 2048 \\
\hline
\end{tabular}

Translator's Note. 'I'Le values given in this table are not thuse of $10-2 \log d$, but merely the complements of the mantissae of $2 \log d$. The object of this procedure is to confine the use of logarithms to additions, eliminating all subtractions.

A calculation, using the example previously given, follows :-

"Rezia." 20th August. $f=322$. Milk diluted 50 times.

3 counts on 3 tubes.

\begin{tabular}{c|c|c|c}
\hline Capillary No. & I & II & III \\
\hline$d=$ & 47 & 49 & 47 \\
\hline 1st reading $a=$ & 124 & 150 & 177 \\
2nd reading $a=$ & 190 & 170 & 153 \\
3rd reading $a=$ & 183 & 194 & 166 \\
\hline Total & 497 & 514 & 496 \\
\hline
\end{tabular}


W. F. Cooper, W. H. Nuttall and G. A. Freak 365

The corresponding value for $10-2 \log d$ is added to the logarithm of each of these three totals:

$\begin{array}{llll}\log 4.97 & 6964 & \log 514 & 7110 \\ 10-2 \log d(47) & 6558 & 10-2 \log d(49) & 6196 \\ \log 225 & 3522 & \log 214 & 3306 \\ & \log 496 & 6868 & \\ 10-2 \log d(47) & \frac{6558}{3516} & \\ & \log 225 & \overline{3516}\end{array}$

The sum of these three figures is taken, $225+214+225=664$. To calculate $N$ from this value, it is only necessary to multiply by the factor given in the table of constants above. Thus, for three readings on three tubes at a dilution of $1: 50$, the factor is 0.5614 , the logarithm of which is 7493. To this is added the logarithm of 664 ,

$\begin{array}{ll}\log 5614 & 7493 \\ \log 664 & 8222 \\ \log \quad 373 & \overline{5715}=\log N .\end{array}$

For the calculation of $V d$, the logarithm of $f(=3 \cdot 22 \%)$ is added to that of $1 \cdot 1$ and the complement of the mantissa of $\log N$ is also added. Thus:

$$
\begin{array}{rr}
\log f & 5079 \\
\log 1 \cdot 1 & 0414
\end{array}
$$

Complement of the mantissa of $\log N(5715) \quad 4285$

$$
\log 9 \cdot 50 \overline{9778}=\log V d \text {. }
$$

In addition to the determination of $N$ and $V d$, the percentage of the globules greater than $2 \cdot 5 \mu$ and $5 \mu$ in diameter were calculated. For this purpose, a special count of the number of globules, exceeding one, and those exceeding two, divisions of the scale was made after each ordinary count.

\begin{tabular}{|c|c|c|c|c|c|c|c|c|c|}
\hline & \multicolumn{3}{|c|}{ Tube No. I, $l=47$} & \multicolumn{3}{|c|}{ 'Tube No. II, $d=49$} & \multicolumn{3}{|c|}{ Tube No. III, $d=47$} \\
\hline & $a$ & $>2.5 \mu$ & $>5 \mu$ & $a$ & $>2 \cdot j \mu$ & $>5 \mu$ & $a$ & $>2 \cdot 5 \mu$ & $>5 \mu$ \\
\hline & & & 4 & & & & 7 & 48 & 4 \\
\hline & 19 & 6 & 5 & 17 & 60 & 4 & 15 & 5 & 5 \\
\hline $3 r d r$ & 183 & (i⿱⺌兀 & 1 & 194 & 87 & 6 & 166 & 74 & 8 \\
\hline Total & 497 & 183 & 13 & 514 & 190 & 16 & 490 & 177 & 17 \\
\hline
\end{tabular}

Observations on the milk of "Rezia," 20th August, will again serve as an example: 


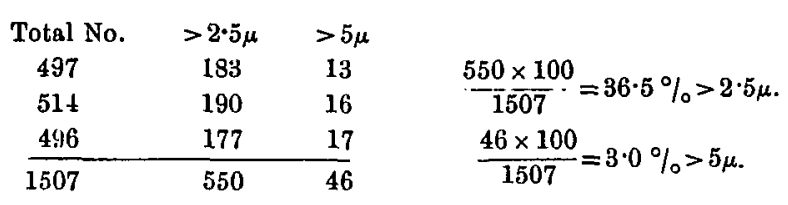

As in counting the globules greater than two divisions we have also included those greater than one, the latter figure must be subtracted from the first to give the percentage required. Thus there are $33.5 \%$ greater than $2 \cdot 5 \mu$,

$$
3.0 \% \quad \text { " } 5 \mu \text {. }
$$

After a little practice, the size of the individual globules can be estimated with a fair degree of accuracy, as shown by the following example.

The figures, employed to demonstrate the accuracy of $V d$, are again used.

1st experiment $N=488 ; \quad V d=10 \cdot 19 \mu^{s} ; 31.31 \%>2.5 \mu, 1.90 \%>5 \mu$. 2nd experiment $N=501 ; V d=9.9 \mu^{3} ; 31.30 \%>2.5 \mu, 1.95 \%>5 \mu$.

Further differentiation between the size of globules is not possible by this method, as, with such great dilutions, there are insufficient globules within the field of the micrometer. If, however, such an estimation appears desirable, the whole of the tube, covered with the slip, may be enumerated.

\section{Flat Cell Method.}

In outline, the method consists in making a photomicrograph of the milk, contained in a Thoma-Zeiss Cell, the enumeration and measurement being carried out on a print.

\section{A. Photomicrography of the Milk.}

The apparatus and method employed are as follows :Apparatus.

(i) A large Zeiss Photomicrographic apparatus is used. This consists of two benches, one supporting the entire optical system-the other the camera. For a full description and illustration of this apparatus, the reader is referred to Zeiss' Catalogue.

The microscope used was a No. 4 projection ocular. The extension of the camera was such as to give a magnification of 500 diameters.

The cell, containing the milk, must be kept in a horizontal position, in order to prevent the fat globules collecting together in one portion 
W. F. Cooper, W. H. Nuttiall and G. A. Freak 367

of it. The microscope is therefore used in a vertical position and a reversing prisin is employed to render the beam horizontal.

(ii) Illumination. This is obtained from a hand-fed arc (carbons at right angles), burning about 13 ampères.

(iii) Exposure. As the fat globules are in continual movement (Brownian), a short exposure is necessary. A Unicum Shutter is interposed in the beam of light, and adjusted to give an exposure of about 1/50 second. The plates used were Paget Extra Special Rapid (Speed about 450 H. and $\mathrm{D}$.), and a normal metol-hydroquinone developer was employed.

(iv) The Cell. The Cell is a specially shallow Thoma blood counting cell, the depth being $0.015 \mathrm{~mm}$. $(15 \mu)$. The area of each square is $1 / 400 \mathrm{sq}$. mm.; so that the side of one square is $0.05 \mathrm{~mm}$. An area of 16 such squares is photographed, so that, at a magnification of 500 diameters, the side of the large square used for counting is

$$
0.05 \times 4 \times 500=100 \mathrm{~mm} \text {. (or about } 4 \text { inches). }
$$

Such a square is conveniently photographed on a half plate.

\section{Method.}

(i) Adjustment of Magnification. The clean empty cell is placed on the microscope stage and adjusted so that an image of the ruling of the squares is projected on to the ground glass screen of the camera which is then extended to give a magnification of exactly 500 diameters.

(ii) Preparation of the Cell. The glass slide and cover slip are both washed with soap and water (on a piece of cotton wool), thoroughly rinsed with water, and dried with a soft cloth.

The sample of unilk is shaken sufficiently to ensure thorough mixing, but care must be taken not to churn it. A small portion is transferred, by means of a platinum loop, on to the centre of the cell, the cover slip is then lowered (by means of a needle) on to the slide and pressed gently, near the edges, till perfect contact is obtained. This is the case when Newton's rings can be seen at the surface of contact of the slide and cover slip. Some practice is necessary to judge the correct quantity of milk to be taken for the cell preparation, but, when the loop has once been adjusted, this is a very simple matter.

(iii) Focussing. In focussing the image on the ground glass screen, some difficulty is encountered, as, owing to the refraction of the light by the globules, no very definite outline can be obtained. Moreover, the globules float up to the underside of the cover-glass, so that the small globules are not in exactly the same plane as the large ones. With practice, however, very good results with sharp outlines can be obtained. 


\section{B. Enumeration of the Fat Globules.}

The distribution of the fat in the various sized globules can be determined from the photographs in two ways:

(i) The total number of globules is determined in a definite area of the photograph-and therefore in a known volume of the milk. This figure, and the percentage of the fat as given by analysis, will give the "mean-diameter" of the globule.

(ii) The diameter of each globule in a definite area of the photograph is measured; and from this, without the aid of any analytical figure, the actual distribution of fat in the various sizes of globules can be computed.

The two methods are detailed below.

\section{Method 1.}

The photomicrographic print, obtained as already described, is ruled to correspond with 16 of the squares on the cell. Thus at 500 diameters magnification, the side of each small square will measure $25 \mathrm{~mm}$., and the side of the square containing 16 of these will be $100 \mathrm{~mm}$.

'The total number of globules in each of the 16 small squares is then counted, each globule being marked off with a pencil as it is recorded; this ensures that each globule is counted once, and once only. The numbers for each of the 16 squares are then added, giving the total number for the whole area. Any globules cutting either of two adjacent sides of the large square (e.g. the top and left hand side) are included; whereas those cutting either of the two remaining sides are neglected.

The percentage of fat in the milk is determined by the centrifuge method.

Since all measurements made on the photograph relate to volumes, and since the analytical result is expressed as $\mathrm{Wt} / \mathrm{Wt}$ percentage, a correction must be applied to correlate the two sets of figures.

Specific gravity of milk fat was taken to be 0.930 . " " milk was taken to be 1.034 .

Then to convert $\mathrm{Wt} / \mathrm{Wt}$ percentage to $\mathrm{Vol} / \mathrm{Vol}$ percentage multiply by

$$
\begin{gathered}
\frac{1 \cdot 034}{\cdot 930}=1 \cdot 1118, \\
{[\log =0 \cdot 0460376] .}
\end{gathered}
$$


W. F. Cooper, W. H. Nuttall and G. A. Freak 369

Volume of the Cell.

16 squares are measured and the area of each square $=\frac{1}{4 \delta 0} \mathrm{~mm} .{ }^{2}$

The depth of the cell is $0.015 \mathrm{~mm}$.

Therefore the volume measured is

$$
\frac{16 \times 0.015}{400} \mathrm{~mm} .{ }^{3}=0.0006 \mathrm{~mm}^{.8}\left(=600,000 \mu^{3}\right) .
$$

Calculation of the "Mean Diameter."

If $d$ and $v$ represent respectively the diameter and volume of a sphere, then

$$
v=\frac{\pi d^{3}}{6} \text { or } d=\sqrt{\frac{6 v}{\pi}} .
$$

Let $f=\%$ of fat (Wt/Wt) found by analysis, and $n=$ No. of globules in the measured volume, then $100 \mu^{3}$ of milk contain $f \times 1 \cdot 1118 \mu^{3}$ of fat.

Therefore $600,000 \mu^{3}$ (the volume measured) contains

$$
f \times 1.1118 \times 6000 \mu^{8} \text { of fat. }
$$

Therefore 1 globule will contain $\frac{1 \cdot 1118 \times 6000}{n} \mu^{3}$ of fat. This represents the volume of the mean sized globule.

Therefore, if $D=$ diameter of the mean globule, then

$$
D=\sqrt[3]{\frac{6 \times f \times 1 \cdot 1118 \times 6000}{n \pi}} .
$$

In practice, logarithms are used for the calculation of $D$. An actnal example will render the method of working quite clear.

\section{Example.}

A sample of milk contained $3.96 \%$ of fat, and the counted volume of the milk contained 1632 globules.

$$
\begin{aligned}
& D=\sqrt[3]{\frac{6 \times 1 \cdot 1118 \times 6000}{\pi} \times \frac{f}{n}}=\sqrt[3]{\frac{6 \times 1 \cdot 1118 \times 6000}{\pi} \times \frac{3.96}{1632}} . \\
& \log 6 \quad=0.7781513 \rightarrow \log \frac{6 \times 1 \cdot 1118 \times 6000}{\pi}=4.1051794 \\
& \begin{array}{l}
\log 1 \cdot 1118=0.0460267 \\
\log 6000=3 \cdot 7781513
\end{array} \quad \text { add } \log 3.96(f) \quad=\frac{0.5976952}{4 \cdot 7028746} \\
& \log 6000=\frac{3 \cdot 7781513}{4 \cdot 6023293} \quad \text { Subtract } \log 1632(n)=3.2127202
\end{aligned}
$$

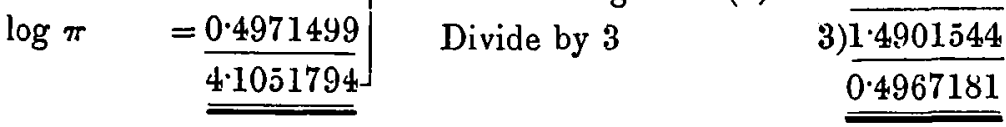

$$
\begin{aligned}
& \text { Hence } D=3 \cdot 14 \mu \text {. } \\
& =\log \text { of } 3 \cdot 1385 \text {. }
\end{aligned}
$$


In calculating a series of such figures, the factor $\frac{6 \times 1 \cdot 1118 \times 6000}{\pi}$ remains constant and so, once obtained, the logarithm of this factor is simply added to that of $f$, the logarithm of $n$ is subtracted and the result divided by 3 to obtain the cube root.

\section{Method 2.}

In this method, not only is a determination made of the number of globules in the ruled area of the photograph, but the diameter of each globule is measured.

For this purpose, a transparency is made of a series of circles, to correspond with $1,2,3, \ldots \mu$ diameter. The magnification of the photographs was 500 diameters, so that the smallest circle was $0.5 \mathrm{~mm}$. or $500 \mu$ diameter; the next $1.0 \mathrm{~mm}$.; $1.5 \mathrm{~mm}$., and so on; corresponding to $1,2,3 \mu \times 500$. This transparency is superposed on the photograph and the diameter of one globule is measured; this is noted and the globule is crossed through with a pencil; another globule is measured, the diameter noted, the globule crossed through; and so on. In this manner, each globule is measured once and once only, and a complete record is obtained of the globules in the counted area. One small square $(25 \times 2.5 \mathrm{cms}$. $)$ is enumerated before proceeding to another, the final result being obtained by the addition of the figures from the 16 squares.

This method of enumeration claims the attention of two workers, one making and calling out the measurements, the other recording them. It is quicker to select all the globules of one size first. After some practice, it is not found necessary to gauge every globule, but only the first few of each size. Since it is impracticable to differentiate between globules of less diameter than $1 \mu$, all such are recorded as $1 \mu$ globules. Moreover, no fraction of $\mu$ is taken into account, but the nearest whole figure is recorded.

From the figures thus obtained, three results may be calculated :

(a) The mean diameter of the globules.

(b) The actual distribution of the fat in the various sizes of globules.

(c) The percentage of fat in the milk.

\section{(a) Calculation of "Mean Diameter."}

The volume of fat contained in a globule of any definite diameter, multiplied by the number of such globules present, gives the volume of 


\section{W. F. Cooper, W. H. Nuttall and G. A. Freak 371}

fat contained by all the globules of that size. 'This operation is repeated for each size of globule in the milk. The sum of these figures gives the total volume of fat present in the measured volume of milk. If the volume, so obtained, be divided by the total number of globules, the volume of the mean sized globule results; from this, it is a simple matter to calculate the diameter of the mean sized globule. The following example shows the method of calculating the volume of the various sized globules ranging from $1 \mu$ to $14 \mu$ in diameter, and also of calculating the diameter of the mean globule.

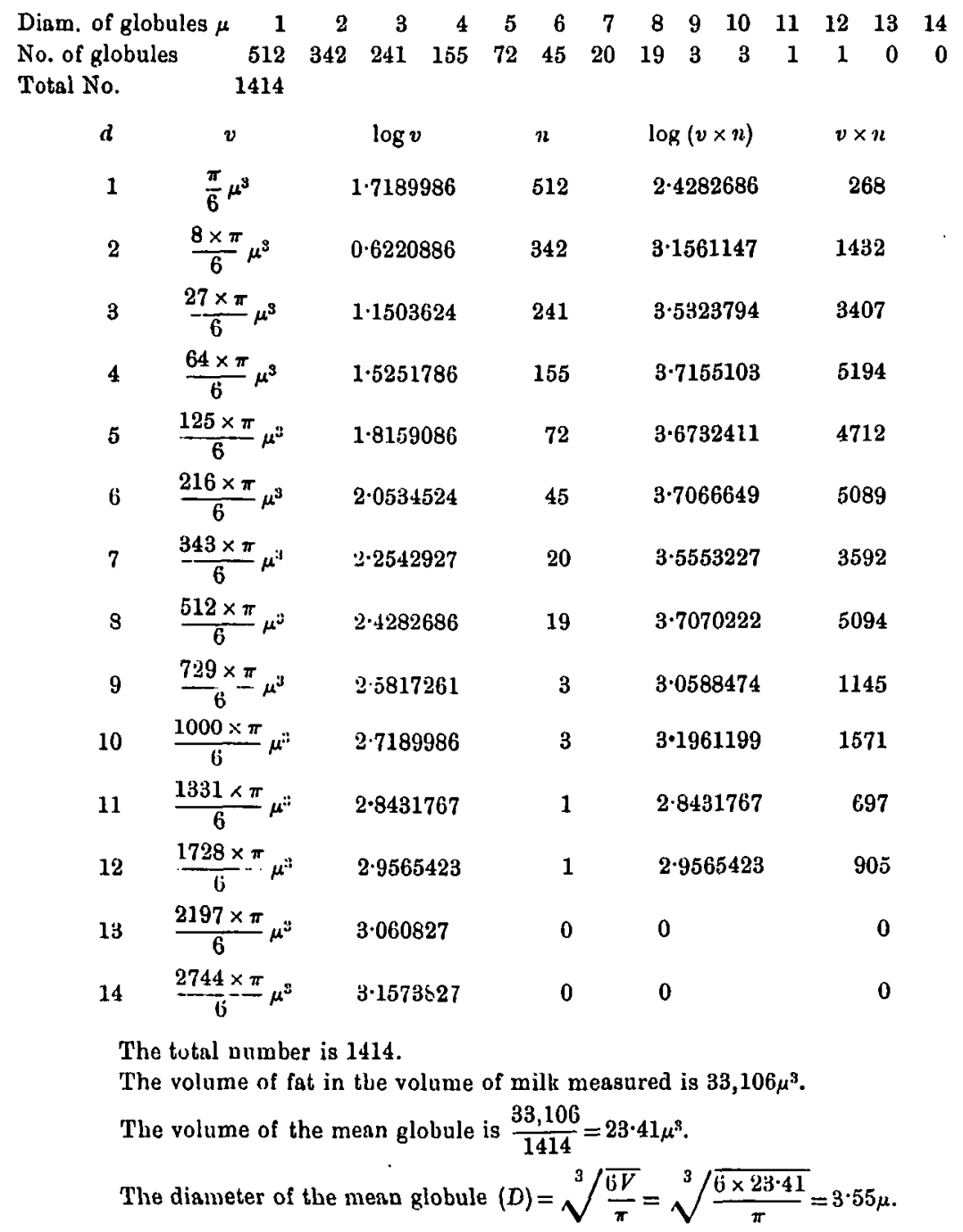


The value for the mean diameter as given by these two methods should be in a fairly close agreement, and where such is not the case, a further examination of the milk can be made. It should be noted that, in Method 1, the percentage of fat determined analytically has been employed; in Method 2, no analytical figure is required, so that if the results, given by the two methods, are in agreement, there is little question of their accuracy.

(b) Distribution of the Fat in the various sized Globules.

Referring to the previous table, it will be seen that the figures under " $v \times n$ " are a measure of the quantity of fat present in any particular size of globule.

For instance, out of a total volume of fat of $33,106 \mu^{3}, 268 \mu^{3}$ is present as $1 \mu$ diameter globules; $1432 \mu^{3}$ as $2 \mu$ diameter globules; and so on. It is a simple matter to transform these numbers into percentages, as has been done in the following table:

$\begin{array}{crc}\begin{array}{c}\text { Diameter } \\ \mu\end{array} & v \times n & \begin{array}{c}\% \text { fat in various } \\ \text { sized globules }\end{array} \\ 1 & 268 & 0 \cdot 81 \\ 2 & 1432 & 4 \cdot 33 \\ 3 & 3407 & 10 \cdot 29 \\ 4 & 5194 & 15 \cdot 69 \\ 5 & 4712 & 14 \cdot 23 \\ 6 & 5089 & 15 \cdot 37 \\ 7 & 3592 & 10 \cdot 85 \\ 8 & 5094 & 15 \cdot 39 \\ 9 & 1145 & 3 \cdot 46 \\ 10 & 1571 & 4 \cdot 74 \\ 11 & 697 & 2 \cdot 11 \\ 12 & 905 & 2 \cdot 73 \\ & \mathbf{3 3 , 1 0 6} & 100 \cdot 00\end{array}$

From the last column, for instance, it will be seen that $15.37 \%$ of the total fat is present in globules of $6 \mu$ diameter; $3.46 \%$ in globules of $9 \mu$ diameter, and so on. A curve, connecting diameter and percentage of fat can be constructed from these figures. In figure 2, such a curve has been drawn $(B)$, together with one showing the relationship between numbers of globules and diameters $(A)$.

The centre of gravity of both curves is indicated. That of curve $B$ represents the true "mean diameter"; that of curve $A$, the "average diameter," i.e. the value which would be arrived at from a purely visual examination, in which only two dimensions can be appreciated. The 
W. F. Cooper, W. H. Nuttall and G. A. Freak 373

large discrepancies between the values for these two centres of gravity is a forcible example of the futility of attempting to estimate the mean diameter by visual examination.

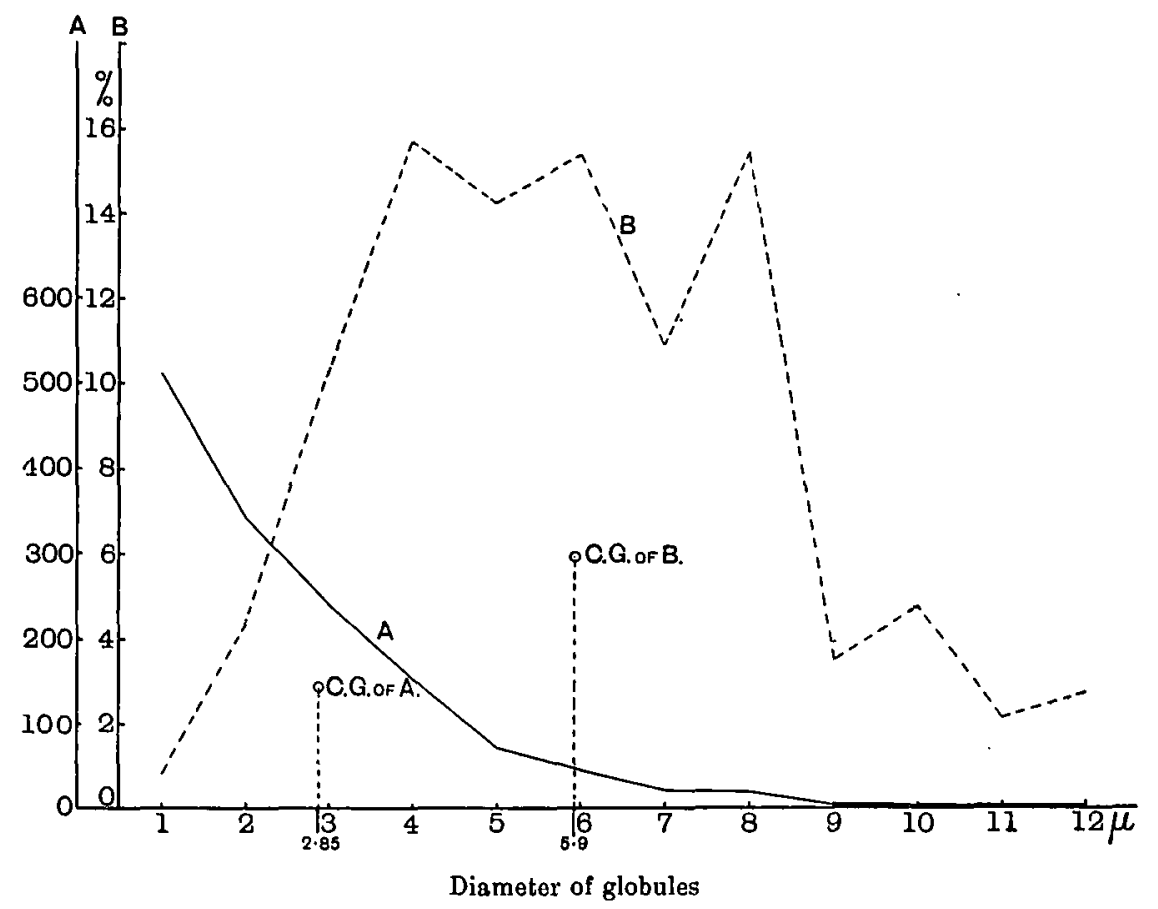

Fig. 2.

(c) The Percentage of Fat in the Milk.

The last column of the table shows that there are $33,106 \mu^{3}$ of fat in the volume of milk measured. This volume has already been shown to be $600,000 \mu^{3}$. Hence the percentage of fat $(\mathrm{Vol} / \mathrm{Vol})$ in the particular sample of milk is

$$
\begin{aligned}
\frac{33,106}{6000} & =5.52 \%(\mathrm{Vol} / \mathrm{Vol}) \\
& =4.96 \%(\mathrm{Wt} / \mathrm{Wt}) .
\end{aligned}
$$

The actual figure furnished by analysis was

$$
4.80 \% \text { fat (Wt/Wt). }
$$

These two figures should be identical, but if the small volume of milk measured (only $0.0006 \mathrm{~mm}^{3}$ ), and the difficulty of accurate measurement of the globules are considered, it will be seen that the agreement is as close as conld be expected. 
If the figure obtained by the above method differs considerably from that obtained by analysis, there is obviously an error in the determination; such an error may occur

(i) in the determination of fat,

(ii) in the selection of the sample for photography,

(iii) in the measurement of the globules.

\section{Degree of accuracy of counting method.}

It was considered desirable to test the reliability of the method, and, to this end, three separate cell preparations and photographs were made on one sample of milk, and three more on another sample. The globules in each of the three photographs were counted, and the resulting figures were compared.

Number of globules in counted area of photograph.

$\begin{array}{ccc}\text { Count } & \text { Sample } A & \text { Sample } B \\ 1 & 2105 & 302 \\ 2 & 2286 & 345 \\ 3 & 2288 & 397 \\ \text { Mean } & 2229 & \overline{348}\end{array}$

The difference between the numbers obtained from the triplicate photographs may be explained by

(i) Errors in sampling.

(ii) Slight difference in focussing the image.

(iii) Errors in counting.

(iv) Lack of "clearness" and density in the print.

(v) Errors due to variation in the volume measured, owing to variation in temperature.

With care, the last three of these possible suurces of error can practically be eliminated; the first is probably the most important, as only $0.0006 \mathrm{~mm}^{3}$ of milk is photographed.

\section{Conclusions.}

Regarding the relative merits of the two methods, the authors have worked with both, and their preference for the cell method is based upon the following considerations.

In Babcock's method, measurements are made upon varying volumes of diluted milk, necessitating the use of a variable factor in the calculation of the mean volume. 
In the cell method, a constant volume of undiluted milk is employed, and hence a constant factor is applied in all calculations. Moreover, the latter method dispenses with the use of an immersing liquid, and accordingly no correction for refraction is necessary.

Again, the volume of milk actually examined in the cell method is roughly ten times that measured in the capillary method.

Whilst several workers have made use of the cell method for milk globule enumerations, the application of photography to it is, as far as the authors are aware, novel. The innovation has several advantages. Foremost amongst these is the fact that permanent records are obtained, which may be enumerated at leisure. Since the film of milk photographed is so thin, no time is wasted waiting for the globules to rise (as is the case in the capillary method), and it is possible to make a very large number of exposures in a short period, the development, printing, couuting, etc., being postponed, if necessary.

In Babcock's method, the effect of Brownian movement upon the smaller globules is liable to introduce two sources of error in counting. Firstly, the number of globules in the counted portion of the capillary is always changing, owing to the small globules at the limits of the measured length constantly leaving and re-entering the enumerated volume. Secondly, this constant oscillatory movement renders the counting of the small globules a matter of uncertainty. Since the calculation of Gutzeit's mean volumes $(V d)$ rests upon the total number of globules counted, and the percentage of fat, it follows that the omission of a minute globule is as serious as that of a very large one.

Gutzeit himself points out that there is a difference of about $30 \%$, in his parallel estimations, so that the above criticism is justified.

The use of a sufficiently short exposure in the photographic method completely obviates this difficulty. The parallel counts given on p. 374 differ by about $14 \%$ respectively - a much better agreement than Gutzeit obtained.

Another very distinct advantage afforded by the photographic method is, that it is possible, by making the double enumeration, as already described (p. 368), to check off the results, and so discard any doubtful values.

In spite of the fact that the method adopted by the authors involves the use of expensive apparatus, and the expenditure of a considerable amount of time, yet they are of the opinion that its advantages far outweigh these objections. It is true that Woll (Agricultural Science, 1892, p. 441) compared the capillary and cell methods and 
concluded in favour of the former. He found that he always detected more globules, when using the capillary, than when using the cell. This is not surprising, as an examination of his technique reveals the fact that in the former case he was using a magnification of 950 diameters, whereas, in the latter, the magnification was only 400 diameters. Gutzeit appears to have accepted Woll's valuation of the reliability of the two methods without question.

A fair comparison of the two methods indicates the distinct superiority of the procedure adopted by the authors. 